\title{
Food Insecurity and Adolescent Psychosocial Adjustment: Indirect Pathways through Caregiver Adjustment and Caregiver-Adolescent Relationship Quality
}

\author{
Beth A. Kotchick $\mathbb{D}^{1} \cdot$ David Whitsett ${ }^{2} \cdot$ Martin F. Sherman ${ }^{1}$
}

Received: 14 September 2020 / Accepted: 17 September 2020 / Published online: 27 September 2020

(c) Springer Science+Business Media, LLC, part of Springer Nature 2020

\begin{abstract}
Household food insecurity is associated with youth behavioral problems, yet few studies have examined potential mechanisms that underline this association, particularly among adolescents. The Family Stress Model (FSM) states that food insecurity potentially impacts adolescent psychosocial adjustment indirectly through its effects on parental psychological functioning and parenting. The current study examined data from the Children, Welfare, and Families study $(N=687,53 \%$ female, $M_{\text {age }}$ of child at baseline $=11.74$ years, $S D=1.39$ ) to determine whether household food insecurity at the beginning of adolescence predicts later behavioral outcomes and whether that association is mediated through caregiver depression and caregiver-adolescent relationship quality. Caregivers completed measures of past-year household food insecurity, current self-reported depressive symptoms and adolescent behavior problems, while adolescents completed a measure of current caregiver-adolescent relationship quality. A serial multiple mediator model, controlling for baseline values of mediators, outcomes, and relevant demographic covariates, indicated a significant total indirect effect, whereas the total direct effect was not significant. Significant indirect effects through both caregiver depression and caregiver-adolescent relationship quality were also found. These results are the first to explicitly examine the FSM with respect to household food insecurity and to demonstrate the indirect effects of food insecurity on adolescent adjustment. The findings indicate the need to improve food security and address subsequent intra- and inter-personal difficulties among low-income families that contribute to behavioral problems among adolescents facing household food insecurity.
\end{abstract}

Keywords Food insecurity $\cdot$ Adolescents $\cdot$ Psychosocial adjustment $\cdot$ Family stress

\section{Introduction}

Food insecurity, which refers to a lack of consistent access to nutritious food due to insufficient financial resources, is a significant stressor faced by $10.5 \%$ of all households in the United States at some time during 2019 (prior to the COVID-19 pandemic), including $13.6 \%$ of all households with youth under the age of 18 years (Coleman-Jensen et al. 2020). Household food insecurity is associated with a number of concurrent emotional and behavioral problems

Beth A. Kotchick

bakotchick@loyola.edu

1 Department of Psychology, Loyola University Maryland, Baltimore, MD, USA

2 Western Youth Services, Fountain Valley, CA, USA among children and adolescents (e.g., Poole-DiSalvo et al. 2016), yet little is known about the processes through which food insecurity may operate on youth psychosocial adjustment or whether those associations are maintained over time. The current study examined the longitudinal association between household food insecurity experienced during early adolescence (ages 10-14) and psychosocial adjustment five years later, as well as the indirect effects of household food insecurity on adolescent psychosocial adjustment through two critical family processes: caregiver-adolescent relationship quality and caregiver depressive symptoms.

\section{Food Insecurity and Youth}

Food insecurity is typically characterized by a number of experiences that reflect difficulty in meeting household food needs, including worrying that food will run out before 
securing funds to purchase more, not being able to afford well-balanced meals, restricting or skipping meals or not eating for an entire day because there isn't enough money to buy food (Coleman-Jensen et al. 2020). More than 35 million individuals live in food-insecure households, including an estimated 10.7 million youth (Coleman-Jensen et al. 2020). Although younger children tend to be somewhat shielded from hunger through access to programs and services such as the National School Lunch Program and The Special Supplemental Nutrition Program for Women, Infants, and Children (WIC; Coleman-Jensen et al. 2020), adolescents are less likely to benefit from such protections and thus shoulder more of the burden of household food insecurity (Moffitt and Ribar 2016). This may occur possibly due to the prioritization of the nutritional needs of younger children within the household and greater social stigma associated with poverty and receipt of food assistance during adolescence that may limit the effectiveness of such interventions for teens (Moffitt and Ribar 2016).

\section{Food Insecurity and Youth Psychosocial Adjustment}

Existing research has established that living in a foodinsecure household is associated with higher rates of behavioral and mental health problems among younger children. For example, preschool-aged children in foodinsecure households demonstrate higher rates of behavioral problems (Whitaker et al. 2006) and developmental delays (Rose-Jacobs et al. 2008), whereas school-aged children who are food insecure tend to show higher rates of depression, anxiety, and externalizing disorders (Slopen et al. 2010) and poorer academic outcomes (Jyoti et al. 2005).

Although the relationship between food insecurity and child psychosocial adjustment is becoming increasingly well-established, research concerning both the immediate and long-term psychosocial impacts of food insecurity among adolescents is relatively sparse. Among adolescents, food insecurity is concurrently associated with behavioral problems (Poole-Di Salvo et al. 2016) and higher rates of suicidal ideation and behavior (Alaimo et al. 2002). However, much of the research concerning the association between household food insecurity and adolescent psychosocial adjustment is limited to cross-sectional analyses, it remains unclear how exposure to household food insecurity during childhood or early adolescence contributes to the risk of psychosocial maladjustment over time. In one exception, an analysis of the same dataset used in the current study revealed that the positive association between household food insecurity experienced between during preand early adolescence (i.e., between 10 and 14 years of age) and elevated rates of internalizing and total behavioral problems persisted into late adolescence (Whitsett et al.
2019). Given that adolescence is a developmental period marked by rapid physical growth and brain development (Abrain et al. 2013), as well as important academic and social transitions (Evans et al. 2018), further research concerning the longitudinal association between household food insecurity experienced during adolescence and psychosocial adjustment, as well as the mechanisms that potentially underlie it, is urgently needed.

\section{The Family Stress Model}

In addition to the direct effects food insecurity may have on behavioral adjustment during adolescence, it is likely that economic stressors, such as food insecurity, may also work indirectly to affect adolescent psychosocial adjustment through the impact they have on the emotional and behavioral functioning of parents (Conger et al. 2010). The Family Stress Model (FSM) states that stress associated with poverty and financial hardship increases parental psychological distress, namely parental depression (Conger et al. 2010), which is consistently related to poorer behavioral outcomes throughout child development (Goodman et al. 2011). Economic stress and parental depression, both independently and in serial, also disrupt effective parenting (e.g., reducing parental warmth, increasing hostile or inconsistent parenting, decreasing monitoring and control over child and adolescent activities; Lee et al. 2011), which is then associated with more internalizing and externalizing problems in both children and adolescents, including higher rates of depression and anxiety (Brumariu and Kerns 2010) and riskier sexual behavior (Kerpelman et al. 2016), and lower levels of self-worth (McAdams et al. 2017). As such, the FSM provides a useful framework for identifying potential mechanisms that could account for the relation between household food insecurity and adolescent psychosocial adjustment.

Empirical support for the FSM is well established for a range of adolescent outcomes and among families of diverse racial and ethnic backgrounds. For example, family economic hardship was indirectly related to externalizing behavior problems and problem drinking through maternal psychological distress and parenting behaviors among an ethnically diverse sample of adolescents (Hardaway and Cornelius 2014). Likewise, the effect of family economic hardship on delinquent behavior among a sample of African American adolescents was mediated by parental depression, caregiver conflict, and negative parenting practices (Simons et al. 2016). Tests of the FSM for food insecurity as a specific indicator of family economic hardship, however, are less common, especially for adolescents.

Some research has examined parent distress and parenting as mediators of the relation between food insecurity and psychosocial adjustment among younger children. For 
example, parental stress, warmth, and responsiveness were found to partially mediate the relationship between household food insecurity and behavioral problems in schoolaged children over a two-year period (Slack and Yoo 2005). Another study found that in a large sample $(N=8944)$ of toddlers, caregiver depression and disrupted parenting practices partially mediated the relationship between food insecurity at 9 months and cognitive development at 24 months (Bronte-Tinkew et al. 2007). Such findings are consistent with the Family Stress Model, as they indicate that caregiver mental health and parenting likely play important roles in the association between food insecurity and child psychosocial adjustment. However, these longitudinal studies only examined younger children and were limited to relatively brief periods of time ( $<2$ years), making it difficult to determine if these indirect effects would be sustained throughout development or whether similar effects would exist for adolescents. Perhaps more importantly, parenting was assessed only from the perspective of caregivers in these studies. As a result, it is possible that method bias may have accounted for a portion of the variance observed in them, particularly if caregivers who were more likely to report food insecurity were also more likely to report more stress, poorer parenting practices, and more child behavioral problems. Still, when considered through the lens of the FSM, these findings would suggest that household food insecurity may indirectly affect adolescent psychosocial adjustment through its detrimental impact on parent mental health, which in turn, would be associated with poorer relationships between parents and adolescents and with less effective parenting practices (Conger et al. 2010).

A more focused examination into whether household food insecurity predicts adolescent psychosocial adjustment through its potential impact on caregivers is significant from a public health perspective, as food insecurity is more than twice as common among adult caregivers as it is among children or adolescents (Coleman-Jensen et al. 2020). If food insecurity impacts adolescent psychosocial adjustment indirectly through its effects on caregivers, adolescents who reside in food insecure households but who are not food insecure themselves may still be at elevated risk for behavioral problems. This would not only suggest that a much larger group of adolescents would be at risk for behavioral problems, but also that interventions that specifically target food insecurity in children and adolescents (e.g., free school lunch programs) may fail to fully ameliorate the detrimental impact of food insecurity on adolescents if they do not also contribute to increased food security at the household level or address caregiver mental health and parenting challenges related to insufficient access to food.

\section{Current Study}

Household food insecurity is consistently associated with poorer psychosocial outcomes in youth at every stage of development, yet the pathways through which food insecurity operates on adolescent psychosocial adjustment remain poorly understood. According to the Family Stress Model, food insecurity may operate on adolescent behavior through its impact on the emotional and behavioral functioning of caregivers, including increasing the risk for caregiver depression and negative parent-adolescent relationships. More specifically, the Family Stress Model would posit that greater food insecurity relates to higher levels of adolescent behavior problems through the negative impact it has on caregiver psychological distress, which in turn, relates to poorer caregiver-adolescent relationship quality, which would then account for poorer adolescent psychosocial adjustment. In the current study, data from the Welfare, Children, and Families survey (Angel et al. 2012), a longitudinal sample of low-income children, adolescents, and their caregivers in the post-welfare reform era, was analyzed to determine whether household food security in early adolescence predicted psychosocial adjustment five years later. As guided by the Family Stress Model, it was hypothesized that household food insecurity during pre- to early adolescence would be positively and directly related to adolescent behavior problems five years later (middle to late adolescence). It was further hypothesized that household food insecurity would predict higher caregiver depressive symptoms and more negative parent-adolescent relationship quality, which in turn, would each predict higher levels of adolescent behavior problems. Moreover, these intervening variables were expected to operate in serial, with household food insecurity being positively associated with greater caregiver depression, which would then be associated with poorer relationship quality and greater adolescent behavior problems. As such, the current study was designed to test whether household food insecurity would have both direct and indirect (through caregiver depressive symptoms and poorer parent-adolescent relationship quality) effects on adolescent psychosocial functioning (see Fig. 1).

\section{Methods}

\section{Participants and Procedure}

Data for the current study were collected as part of The Welfare, Children, and Families Study (WCFS; Angel et al. 2012). The WCFS evaluated the health and well-being of 


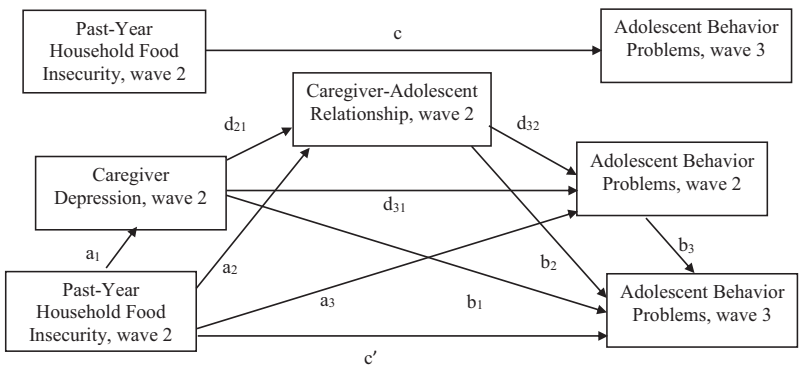

Fig. 1 Hypothesized serial multiple mediation model of household food insecurity effects on adolescent total behavior problems with caregiver depression and caregiver-adolescent relationship quality as proposed mediators

low-income families in three different cities in the years following welfare reform (1999-2005). The full sample included 2402 households in Boston, Chicago and San Antonio, all of whom had at least one child and lived below $200 \%$ of the poverty line. Data were collected among census blocks in each city that had a high number of residents living below the poverty line, and families were selected for participation within these blocks using stratified random sampling. Data collection took place at three points in time. The first wave (baseline) of interviews with the primary female caregiver (referred to as caregiver throughout this paper) were conducted in 1999 when the focal child was either between 0 and 4 or between 10 and 14 years of age. Wave 2 of the study was completed when the focal child in each household was between 1 and 6 years old or 11 and 16 years old (2000), and wave 3 was completed when the same child was between 5 and 10 or 15 and 20 years old (2005). More detailed information on the study design can be found through the online resource database provided as a part of the Welfare, Children, and Families Study (Angel et al. 2012).

The current analysis included households with focal children between 10 and 14 years who identified as nonHispanic white, non-Hispanic black, or non-white Hispanic and who completed both child and caregiver rating forms $(N=1150)$. Because the focus of the current study was on longitudinal effects of food insecurity on adolescent psychosocial adjustment, only cases where caregivers were present to complete adolescent behavior problems ratings for all three time points across the five year study span $(n=$ $715 ; 62 \%$ retention from baseline) were included. An additional 28 cases were deleted due to missing values on key demographic variables that were statistical covariates for the primary analyses, yielding a final sample of 687 households (60\% retention from baseline). Within the final sample, the majority of caregivers were unmarried (84\%) and reported their ethnicity as non-white Hispanic (46\%) or African American (43\%). Caregiver age ranged from 18 to 74 years, with a mean age of 38.20 years $(S D=8.25)$.
Table 1 Descriptive statistics for variables used in the analysis at baseline (Wave 1), final sample $(N=687)$

\begin{tabular}{lll}
\hline Variables & $\begin{array}{l}\text { Mean or } \\
\text { frequency }\end{array}$ & $S D$ \\
\hline Predictor & & \\
$\quad$ Food insecurity & 0.41 & 1.19 \\
Outcomes & & 16.63 \\
$\quad$ Behavioral problems (raw scores) & 21.82 & \\
Mediators & & 0.70 \\
Caregiver depression & 1.51 & 0.70 \\
Caregiver-adolescent relationship & 3.91 & \\
$\quad$ quality & & \\
Covariates & & \\
Child sex & & \\
$\quad$ Male & $47 \%$ & \\
$\quad$ Female & $53 \%$ & \\
Child age & 11.74 & \\
Caregiver Race & & \\
$\quad$ White & $10 \%$ & \\
$\quad$ Black & & \\
$\quad$ Hispanic & & \\
Caregiver age (years) & & \\
Number of other children in household & 1.92 & \\
Number of adults in household & 1.56 & \\
Below federal poverty line & $73 \%$ & \\
Income-to-needs ratio & 0.76 & \\
Access to emergency funds & $33 \%$ & \\
Married & $16 \%$ & \\
Caregiver has high school diploma & $51 \%$ & \\
Doighborhood problems & 20.14 & \\
\hline
\end{tabular}

Approximately half of caregiver respondents had a high school diploma or higher (51\%). Domestic violence was somewhat common, with $27 \%$ of respondents reporting having experienced at least one incidence of domestic violence in the year prior to the baseline interview. The mean age of all focal children at baseline was 11.74 years $(S D=1.39)$, with the majority being female $(53 \%)$. More than $73 \%$ of all families were below the official 1999 federal poverty line (i.e., falling below an income-to-needs level of 1), and all families were below twice the official poverty line (i.e., falling below an income-to-needs level of 2). Demographic characteristics for the final sample are summarized in Table 1.

Baseline interviews were completed with the focal child and the caregiver in the household and lasted approximately two and a half hours in length. After completion of the first wave (1999-2000), interviews were repeated in two subsequent waves (wave 2 in 2000 and wave 3 in 2005). Surveys were conducted in-person by a trained interviewer 
and completed in either English or Spanish using Automated Computer Assisted Survey Interview methodology (ACASI). Adult participants were paid a $\$ 30$ incentive upon the completion of each survey. The dataset is publicly available and was downloaded from the Welfare, Children, and Families website (Angel et al. 2012). For more detailed information regarding data collection procedures, see Angel et al. (2012) for a complete summary of the study design and procedures. The study received IRB approval from all universities and data collection firms involved in the project (Angel et al. 2012).

\section{Measures}

\section{Food insecurity}

The main predictor of interest was household food insecurity over the 12 months prior to the wave 2 interview, which was evaluated retrospectively using an abbreviated version of the 18-item USDA Household Food Security Module (HFSS; Bickel et al. 2000). The full HFSS module serves as the "gold standard" (Hager et al. 2010) for measuring past-year household insecurity (Cook et al. 2013). The items within the module are ranked sequentially in order of severity of food insecurity on a 0-10 scale over the past twelve months, from least ("respondent worried food would run out") to most severe ("child did not eat for a whole day"). The total number of affirmative responses on the HFSS module provides a continuous measure of each household's food insecurity, which is then classified into one of four categories of food security: High Food Security, Marginal Food Security, Low Food Security, Very Low Food Security. It should be noted that even marginal household food insecurity ( 1 or 2 reported items) is associated with increased health risks (Cook et al. 2013).

In lieu of the full 18-item HFSS module, the WCFS utilized an abbreviated version consisting of eight items, all of which were calibrated to exceed minimal severity thresholds for a classification of "food insecure" (Low Food Security or Very Low Food Security, according to United States Department of Agriculture 2018). The primary caregiver completed the measure at each wave of data collection. This provided a measure of household food insecurity during the previous year (when focal children were between 10 and 14 years of age). For the purpose of the current analyses, affirmative responses on these 8 items were summed to produce a continuous measure of household food insecurity, with higher totals indicating greater food insecurity during the past year. Several studies indicate that responses to the full 18 -item HFSS provide reliable and valid assessment of food insecurity (Hamilton et al. 1997), and responses to the modified eight-item HFSS scale used in the WCFS showed internal consistency within the current sample (alpha $=0.81)$.

\section{Adolescent behavior problems}

The primary outcome of interest was adolescent psychosocial adjustment, which was operationally defined as behavior problems evaluated at all three waves of data collection (when children were between 10 to 14 years old, 11 to 16 years old, and 16 to 20 years old, respectively). Behavior problems were assessed using 113-item Achenbach Child Behavior Checklist (CBCL 4-18; Achenbach 1991; CBCL 6-18; Achenbach and Rescorla 2001) for focal children under the age of 18 at the time of interview. The same primary caregiver completed the measure at each wave of data collection. The CBCL 4/18 was administered at Waves 1 and 2, and either the CBCL 6/18, an updated version of the CBCL/4-18 that included new age-based norms and six replacement items (\#2, 4, 5, 28, 78, 99), or the ABCL, a scale that assesses behavior problems for individuals ages 18 or older, was administered at Wave 3 . Both versions of the CBCL contain 113 items that are rated as Not True (0), Somewhat or Sometimes True (1), or Very True or Often True (2). The ABCL contains 126-items that are also rated as Not True (0), Somewhat or Sometimes True (1), or Very True or Often True (2). The three measures are strongly and positively correlated, suggesting that they assess the same underlying constructs (Achenbach and Rescorla 2001). On both versions of the CBCL and the $\mathrm{ABCL}$, items are clustered to form scales that measure internalizing problems (anxious, depressed, withdrawn) and externalizing problems (aggressive, rule-breaking) (Achenbach 1991, 1997; Achenbach and Rescorla 2001) and a total problems score is computed by summing the raw scores of all problem items, with higher scores reflecting greater problems (and poorer psychosocial adjustment). Raw scores can also be converted to standardized T-scores normed by age and gender developed from a large, nationally representative sample of children. In keeping with the authors' recommendations (Achenbach 1991; Achenbach and Rescorla 2001), however, only raw scores were used for the primary data analysis. The percentage of children that were missing at least one item from the $\mathrm{CBCL}$ and the $\mathrm{ABCL}$ across waves 2 and 3 averaged $9.13 \%$. The average percentage of missing values across all children and across the total number of potential data values was only $0.21 \%$ (ranging from a low of $0.13 \%$ to a high of $0.32 \%$ ). Multiple imputation was used to handle missing values given its advantage of providing unbiased estimates and utilizing all available data which preserves sample size and statistical power (McCleary 2002). SPSS26.0 was used to scan the data to determine the best imputation method in order to generate ten imputed data sets. The ten data sets were 
then aggregated utilizing the mean to form one pooled data set.

Responses to items on 113-item Total Problems on CBCL/4-18, CBCL scale/6-18, and ABCL scale have demonstrated both internal consistency and test-retest reliability across a wide range of ages (Achenbach 1991, 1997; Achenbach and Rescorla 2001). In order to ensure consistency across the slightly different scales used for different time points and different ages, the total behavior problems scale used for data analysis was calculated using only the 69 items that were identically-worded across all three scales. Scores from the modified, 69-item total problems scale from the initial sample from the WCFS demonstrated high internal consistency for Total Problems at wave 1 (alpha $=0.94)$, wave 2 (alpha $=0.94)$, and wave 3 (alpha $=0.94)$. Scores at wave 1 were highly correlated with scores at wave $2(r(685)=0.59, p<0.001)$ and at wave $3(r(685)=0.48, p<0.001)$. Scores on the modified 69 -item scale were highly correlated with the full 112-item CBCL scales at wave $1(\mathrm{r}(685)=0.99, p<0.001)$, wave 2 $(r \quad(685=0.99, \quad p<0.001)$, wave $3 \quad(r \quad(498)=0.99$, $p<0.001)$, and 126 ABCL-item scales at wave $3(r(185)=$ $0.97, p<0.001)$.

With reference to the validity of item responses, previous studies have shown that Total Problems scores discriminate $(p<0.01)$ between clinically referred and non-referred children, with referral status accounting for approximately $36 \%$ of covariance in Total Problems scores. Referred children also were much more likely to obtain borderline or clinically elevated scores $(\mathrm{T}>60)$ on the scale than nonreferred children (Achenbach 1991; Achenbach and Rescorla 2001).

\section{Caregiver depression}

The depression subscale from the Brief Symptom Inventory-18 (BSI-18; Derogatis 2000) was used to measure caregiver depression at wave 2 . The BSI-18 is an 18item measure of general psychological stress. The depression subscale consists of six items. On this subscale, participants are asked to rate how much they have been "distressed or bothered" in the past 7 days by common depression symptoms (e.g., "feeling of worthlessness", "feeling hopeless about the future"). Responses are based on a 5-point Likert-type scale that ranges from 0 (not at all) to 4 (extremely) for a possible range of 0-24 for the total subscale score, with higher scores reflecting greater symptoms of depression that are considered to be distressing or bothersome. Responses to the depression subscale have shown good internal consistency in other samples of US adults (Derogatis 2000), as well as good test-retest reliability over a 15-day period (Andreu et al. 2008). Depression subscale scores on the BSI-18 are associated with scores on other depression subscales including those of the BDI $(r=0.83 ; p<0.001)$ and MMPI-2 Depression score ( $r=0.78 ; p<0.001$; Andreu et al. 2008). In the original sample of participants from the Welfare, Children, and Families Study, responses on the subscale showed high internal consistency at wave 1 (alpha $=0.85)$ and wave 2 $($ alpha $=0.87)$. The percentage of caregivers that were missing at least one item from the BSI depression subscale was $0.66 \%$ and the percentage of missing data values across all caregivers and across all BSI depression items was $0.34 \%$. SPSS 26.0 was again used to scan the data to determine the best imputation method. Ten imputed data sets were then generated and pooled by averaging to form the final data set.

\section{Caregiver-adolescent relationship quality}

An abbreviated version of the parent version of the Inventory of Parent and Peer Attachment (IPPA; Armsden and Greenberg 1987) was used to assess overall relationship quality within the caregiver-adolescent relationship. The scale was completed by the focal child at wave 2 . The original version of the IPPA consisted of 28 items that assess both the affective and cognitive aspects of adolescents' attachment to their parents or caregivers (e.g., "my mother accepts me as I am"); respondents are required to rate the degree to which each item is true for them on a fivepoint scale ranging from "Almost always or always true" to "Almost never or never true". The items in the original scale are divided into three subscales that reflect different aspects of the attachment relationship (trust, communication, and anger/alienation). Negatively worded items are reverse scored and items are summed to produce subscale scores; all items can also be summed together to create a global score of the overall quality of attachment, with higher scores indicating a more positive relationship between parent and adolescent. The full version of the parent IPPA scale has shown adequate internal consistency for subscale and total scores (Armsden and Greenberg 1987) and validity, with lower scores on the IPPA scales being significantly associated with higher levels of adolescent clinical depression and anxiety (Papini and Roggman 1992) and antisocial behavior (Marcus and Betzer 1996).

The abbreviated scale developed for use in the WCFS consisted of 12 items selected from the full IPPA scale based on the theoretical strength of their relationship to the construct of caregiver-adolescent relationship quality. Principal component factor analysis was then used to statistically determine the constructs that best reflected overall quality of the caregiver-adolescent relationship, yielding a two-factor solution of trust/communication and anger/alienation (Angel et al. 2012). The resulting subscales (trust/ communication and anger/alienation) contain six items 
each. After reverse-scoring the anger/alienation items, the mean of all 12 items is calculated to form an overall measure of caregiver-adolescent relationship quality, with higher scores indicating better relationship quality. The responses to the abbreviated scale's items in the original sample of adolescents in the Welfare, Children, and Families Study showed adequate internal consistency at wave 1 (alpha $=0.73$ ) and wave 2 (alpha $=0.82)$. The percentage of respondents missing at least one item on the abbreviated IPPA was $2.34 \%$ and the percentage of missing data values across all respondents and across all IPPA items was $0.33 \%$. Again, SPSS 26.0 was used to scan the data to determine the best imputation method. Ten imputed data sets were then generated and pooled by averaging to form one final data set.

\section{Covariates}

Several factors assessed in the WCFS that have been shown to be associated with the predictor of interest (household food insecurity), mediators (caregiver depression and caregiver-adolescent relationship quality) and/or outcomes (adolescent behavior problems) were included as covariates in the primary analyses. These included age of the primary female caregiver (e.g., Tearne 2015) at baseline, child age and sex (e.g., Ramtekkar et al. 2010), caregiver ethnicity (two dummy variables were created to categorize caregiver ethnicity into three descriptors: White, Black, and nonWhite Hispanic; e.g., Myers and Painter 2017), caregiver education (did not earn high school diploma or earned high school diploma; e.g., Harding 2015), caregiver marital status (unmarried or married; e.g., Daryani et al. 2016), number of other adults living in the household (e.g., Burchinal et al. 1996), and number of other children living in the household (e.g., Fox et al. 1995). Given prior studies that suggest a strong association between domestic violence and household food insecurity (e.g., Munger et al. 2016), a measure of domestic violence experienced by the caregiver in the previous 12 months (did not experience domestic violence or experienced domestic violence) was also included in all models. The ratio between household income and number of people in the household was used as an indicator of overall poverty status in order to account for likely covariance between overall economic hardship and household food insecurity across time. Ratios of 1 reflect a family existing at the official definition of poverty, ratios $<1$ reflect a family existing below the poverty level, and ratios $>1$ reflect a family existing above the poverty level (e.g., a ratio value of 1.25 indicates that income was $125 \%$ above the appropriate poverty threshold). As research suggests that caregivers' access to instrumental supports through one's social network acts as a buffer against child behavior problems in low-income families (Ryan et al. 2009), a binary measure of each household's perceived access to emergency financial resources ("when I need someone to loan me money in an emergency, I have no one or too few people to count on, or enough people to count on") was included. To account for neighborhood effects (Minh et al. 2017), a measure of self-reported neighborhood problems was also included in all models. Self-reported neighborhood problems were measured using an 11-item scale that was developed by the Welfare, Children, and Families Study investigators to assess respondents' perceptions of problems (e.g., assaults and muggings, drug dealing, gangs, abandoned houses, and teenage pregnancy) in their local neighborhood. For each problem, participants responded using a Likert-type response format ranging from 1 "Not a Problem" to 3 "A Big Problem"); scores could range from 11 to 33 with higher scores reflecting a greater severity of neighborhood problems. In the full WCFS sample, responses to the neighborhood problems scale yield an alpha of 0.91 in wave 1 .

\section{Statistical Analysis Overview}

Bivariate correlations between household food insecurity, caregiver depression, caregiver-adolescent relationship quality, and adolescent behavior problems were examined using Pearson correlation coefficients (see Table 2). Bootstrapping was utilized to estimate the indirect effects of household food insecurity on future behavior problems via caregiver depression and caregiver-adolescent relationship quality in serial, using 10,000 bootstrapping samples for each model (Preacher and Hayes 2008). Serial multiple mediator models were generated using Hayes' PROCESS macro (Hayes 2012), specifying model 6. First, the unadjusted relationships between household food insecurity from the wave 2 interview were estimated; specifically, this model estimated the path between food insecurity during the 12 months prior to the interview (when the focal child was aged 10-14 years) and total behavioral problems at wave 3 (when the focal child aged 15-18 years). The three mediators of interest (caregiver depression, caregiver-adolescent relationship quality, and adolescent behavior problems, all assessed at the time of the wave 2 interview) were then entered into the model, with caregiver depression being entered first in the sequence, followed by relationship quality and adolescent behavior problems, respectively. Baseline scores for all three mediators were entered as covariates to strengthen the causal argument between wave 2 food insecurity and the three wave 2 mediators of interest. Similarly, the model adjusted for wave 2 behavior problems when estimating the association between the two caregiver-level mediators (wave 2 caregiver depression and relationship quality) and wave 3 adolescent behavior problems in order to strengthen the 
Table 2 Means, standard deviations, and zero-order variables correlations among research

\begin{tabular}{|c|c|c|c|c|c|c|}
\hline & 1. & 2. & 3. & 4. & 5. & 6. \\
\hline 1. Food insecurity & - & - & - & - & - & - \\
\hline 2. Caregiver depression & $0.21 * * *$ & - & - & - & - & - \\
\hline 3. Relationship quality & $-0.11 * *$ & $-0.14 * * *$ & - & - & - & - \\
\hline $\begin{array}{l}\text { 4. Behavior problems } \\
\text { (Wave 1) }\end{array}$ & $0.21 * * *$ & $0.39 * * *$ & $-0.16^{* * *}$ & - & - & - \\
\hline $\begin{array}{l}\text { 5. Behavior problems } \\
\text { (Wave 2) }\end{array}$ & $0.26 * * *$ & $0.40 * * *$ & $-0.18 * * *$ & $0.59 * * *$ & - & - \\
\hline $\begin{array}{l}\text { 6.Behavior problems } \\
\text { (Wave 3) }\end{array}$ & $0.14 * * *$ & $0.29 * * *$ & $-0.20 * * *$ & $0.48 * * *$ & $0.50 * *$ & - \\
\hline Mean & 0.41 & 1.51 & 3.91 & 21.82 & 18.88 & 20.07 \\
\hline$S D$ & 1.19 & 0.70 & 0.70 & 16.63 & 15.39 & 16.83 \\
\hline Range & $0-8$ & $1-5$ & $1-5$ & $0-89$ & $0-81$ & $0-79$ \\
\hline
\end{tabular}

causal argument for last step of the pathway. All models also included covariates assessed during the study's first wave, when the focal child was 10-14 years old, to statistically control for the associations between those variables and the mediators and outcome variables in the primary analyses. Wave 1 covariates were considered most appropriate, as they roughly approximated the beginning of the twelve-month span over which wave 2 food insecurity was retroactively evaluated. All analyses were performed using SPSS v. 24 statistic software.

\section{Results}

\section{Preliminary and Descriptive Analyses}

The mean level of household food insecurity in the year prior to the interview was $0.41(S D=1.19$; range: $0-8)$, with $15 \%$ $(N=100)$ of adolescents living in a household that provided at least one affirmative response to the food insecurity items. At baseline, average raw total problem behavior scores for the final sample were $21.82(S D=16.63)$, with skewness of 1.14 $(S E=0.09)$. Average caregiver depression scores at wave 2 were $1.50(S D=0.70)$, with skewness of $2.21(S E=0.09)$; caregiver-child relationship quality scores at wave 2 were $3.91(S D=0.70)$, with skewness of $-0.84(S E=0.09)$; average raw total problem behavior scores at wave 2 were $18.88(S D=15.39)$, with skewness of $1.19(S E=0.09)$; and average raw total problem behavior scores at wave 3 were $20.07(S D=16.83)$, with skewness of $1.11(S E=0.09)$. There was a significant positive relation between household food insecurity scores and caregiver depression, $r(685)=$ $0.14, p<0.001$, and between household food insecurity and total behavior problems, $r(685)=0.21, p<0.001$. As noted earlier, there was a loss of some participants due to attrition $(n=435)$ and incomplete demographic data $(n=28)$. One-

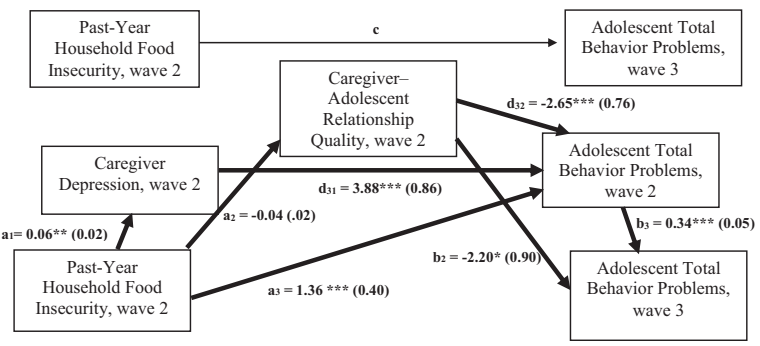

Fig. 2 Results of serial multiple mediation model: total behavioral problems ${ }^{\dagger}$. Only total, direct, and indirect effect pathways with $p<$ 0.05 are shown. Standard errors are presented in parentheses. ${ }^{*} p<$ $0.05 ; * * p<0.01 ; * * * p<0.001$

way ANOVA tests did not reveal any significant differences between those who were and were not included in the current analyses across any demographic variable included in this study. There was no significant relation between the odds of dropout and household food insecurity scores, caregiver depression, caregiver-adolescent relationship, or total behavior problem scores.

\section{Primary Analyses}

For the primary analysis, a serial multiple mediator mediation model (model 6) was used to test the hypothesis that household food insecurity experienced during early adolescence would be predictive of future total adolescent problem behaviors directly and indirectly through caregiver depression and caregiver-adolescent relationship quality in sequence; all path estimates were generated using non-parametric bootstrapping methods (Hayes 2018). All significant paths are represented in Fig. 2, with coefficients for all paths listed in Table 3. The total direct effect was not significant $\left(c^{\prime} ; B=-0.21,95 \%\right.$ CI $\left.[-1.14,0.71]\right)$, but the total indirect effect (i.e., the sum of all specific indirect effects) was significant $(B=0.64,95 \%$ CI $[0.25,1.16])$. As hypothesized, the 
Table 3 Path coefficients, direct effects, and indirect effects for total behavior problems from Fig. 1

\begin{tabular}{lcll}
\hline Path & $b$ & \multicolumn{2}{l}{$S E$} \\
\hline a1 & $0.06^{* *}$ & 0.02 & \\
a2 & -0.04 & 0.02 & \\
a3 & $1.36^{* * *}$ & 0.40 & \\
b1 & -0.40 & 1.03 & \\
b2 & $-2.20^{*}$ & 0.90 & \\
b3 & $0.34^{* * *}$ & 0.05 & \\
d21 & -0.06 & 0.04 & \\
d31 & $3.88^{* * *}$ & 0.86 & \\
d32 & $-2.65^{* * *}$ & 0.76 & \\
& & & \\
Summary of Significant Indirect & $b$ & $S E$ & $95 \% \mathrm{CI}$ \\
Effects & & & \\
\hline Total indirect effect & & & \\
Indirect effect $\left(\mathrm{a}_{1} \times \mathrm{d}_{31} \times \mathrm{b}_{3}\right)$ & 0.04 & 0.04 & $0.25-1.14$ \\
Indirect effect $\left(\mathrm{a}_{2} \times \mathrm{b}_{2}\right)$ & 0.08 & 0.06 & $0.01-0.26$ \\
Indirect effect $\left(\mathrm{a}_{2} \times \mathrm{d}_{32} \times \mathrm{b}_{3}\right)$ & 0.03 & 0.02 & $0.00-0.10$ \\
Indirect effect $\left(\mathrm{a}_{3} \times \mathrm{b}_{3}\right)$ & 0.46 & 0.18 & $0.16-0.88$ \\
\hline
\end{tabular}

$N=687$

$* p<0.05 ; * * p<0.01 ; * * * p<0.001$

specific indirect effect through caregiver depression and adolescent behavior problems in serial at wave 2 was significant $\left(\left(a_{1} \times d_{31} \times b_{3}\right)=0.08\right.$; $\left.\mathrm{CI}=0.02-0.18\right)$, as was the effect of household food insecurity through adolescent behavior problems at wave $2\left(a 3^{*} b 3=0.46 ; \quad \mathrm{CI}=\right.$ $0.17-0.88)$. The specific indirect effect through poorer caregiver-adolescent relationship quality at wave 2 was also significant $(a 2 \times b 2=0.08 ; \mathrm{CI}=0.01-0.26)$, as was the indirect effect of food insecurity through poorer caregiver-adolescent relationship quality at wave 2 and adolescent behavior problems by wave 2 in serial $\left(a 2 \times d_{32} \times b 3\right.$ $=0.03 ; \mathrm{CI}=0.01-0.10)$. The hypothesized serial indirect effect of food insecurity through caregiver depression operating on caregiver-adolescent relationship quality was not significant.

To examine the robustness of these estimates, three alternative models with different model specifications were tested. First, household food insecurity was treated as a binary variable, either food secure $(n=587)$ or food insecure $(n=$ 100). The designation of food insecure was assigned when there was a positive response on any one of the eight food insecurity questions, an approach that is consistent with prior use of other abbreviated or screening versions of the full HFSS module in which an affirmative response to single item is considered a marker for food insecurity (Coleman-Jensen et al. 2020). This alternative statistical model demonstrated similar patterns of results as original model. For total scores, the total direct effect was not significant $\left(c^{\prime}\right)(B=0.32,95 \%$
CI $[-2.79,3.44])$, and the total indirect effect was still significant $(B=2.20,95 \%$ CI $[1.03,3.83])$. The specific indirect effect through caregiver depression and adolescent behavior problems in serial at wave 2 was again significant $\left(\left(a_{1} \times d_{31} \times\right.\right.$ $\left.b_{3}\right)=0.26 ; \mathrm{CI}=0.08-0.63$ ), as was the effect of household food insecurity through child behavior problems by wave 2 $(a 3 \times b 3=1.63 ; \mathrm{CI}=0.62-3.10)$. The specific indirect effect through caregiver-child relationship quality again was significant $(a 2 \times b 2=0.26 ; \mathrm{CI}=0.01-0.84)$, as was the indirect effect of food insecurity through caregiver-child relationship quality at wave 2 and child behavior problems by wave 2 in serial $\left(a 2 \times d_{32} \times b 3=0.11 ; \mathrm{CI}=0.01-0.32\right)$.

Second, the hypothesized model was tested using a binary outcome variable which indicated whether adolescent behavior problems were above or below the threshold for clinical significance using standardized scores on the original full CBCL or ABCL measures. At baseline, almost a third $(n=$ $225)$ of participants were above the threshold for clinical significance (T-score $>60)$. This decreased slightly as focal children aged, as the number of clinically-elevated participants had decreased by wave $2(n=185)$ and wave $3(n=$ 183). This alternative approach again demonstrated similar patterns of results in that the total direct effect was not significant $\left(c^{\prime}\right)(B=-0.06,95 \%$ CI $[-0.23,0.11])$ and the total indirect effect was significant $(B=0.09,95 \%$ CI $[0.03$, $0.18])$. The specific indirect effect through caregiver depression and adolescent behavior problems in serial at wave 2 remained significant $\left(\left(a_{1} \times d_{31} \times b_{3}\right)=0.01 ; \mathrm{CI}=0.00-0.02\right)$, the effect of household food insecurity through child behavior problems by wave 2 remained significant $(a 3 \times b 3=0.06$; $\mathrm{CI}=0.02-0.12$ ), and the specific indirect effect through caregiver-child relationship quality again was significant $(a 2 \times b 2=0.01 ; \mathrm{CI}=0.00-0.05)$.

Lastly, as the original models suggested the presence of independent, parallel pathways through caregiver depression and relationship quality, respectively, a parallel mediation model was estimated with caregiver depression, caregiver-child relationship quality, and child behavioral problems at wave 2 serving as independent mediators (e.g., not in serial). The total direct effect was again not significant, but the total indirect effect (i.e., the sum of all specific indirect effects) was significant $(B=0.64,95 \%$ CI $[0.24,1.14])$. The effect of household food insecurity through caregiver-child relationship quality at wave 2 was again significant, with a slightly larger effect size than in the serial model $(a 1 \times b 1=0.09 ; \mathrm{CI}=0.01-0.28)$. As predicted, the specific indirect effect through caregiver depression at wave 2 was not significant $(a 2 \times b 2=-0.02$; $\mathrm{CI}=-0.21$ to 0.13 ), as this pathway operated in serial through child behavioral problems at wave 2 in the original model. The specific indirect effect through child behavioral problems at wave 2 was again significant using the parallel mediation model $(a 3 \times b 3=0.58 ; \mathrm{CI}=0.23-1.01)$. 


\section{Discussion}

Household food insecurity is a stressor faced by over 10 million youth (Coleman-Jensen et al. 2020) and which carries significant risk for psychological maladjustment in the form of both internalizing (e.g., depression, anxiety) and externalizing (e.g., aggression, conduct problems) symptoms among children (e.g., Slopen et al. 2010) and adolescents (e.g., Poole-Di Salvo et al. 2016). Yet the mechanisms through with household food insecurity operates on youth psychosocial adjustment and how those effects manifest over time are poorly understood, particularly among adolescents. The Family Stress Model (Conger et al. 2010) offers a framework to understand how economic hardship, such as food insecurity, might have both direct and indirect effects on youth psychosocial functioning through caregiver depression and caregiver-adolescent relationship quality. The current study used data from the Welfare, Children, and Families Study (Angel et al. 2012) to examine whether this model could predict behavior problems among middle- to late-adolescents who had been exposed to household food insecurity during pre- or early adolescence.

The current findings indicated that household food insecurity reported by caregivers as occurring over the previous 12 months had both direct and indirect longitudinal effects on adolescent behavior problems. Specifically, caregiver-reported household food insecurity was associated with higher levels of caregiver depressive symptoms and poorer quality adolescent-caregiver relationships. These variables were, in turn, associated with significantly greater behavioral problems among adolescents approximately five years later. This finding is consistent with the Family Stress Model, which posits that socioeconomic hardships operate through parental behavior to impact child and adolescent development. As such, it is speculated that household food insecurity is a chronic source of stress that potentially contributes to caregiver depressive symptoms and negative patterns of caregiver-child interaction over time (Conger et al. 2010). These, in turn, potentially lead to stress-sensitivity, reactivity and behavior problems among youth in the household which persist throughout the entire period of adolescence (e.g., Hardaway and Cornelius 2014; Simons et al. 2016).

Importantly, the Family Stress Model would posit that parental or caregiver psychological distress, measured as depressive symptoms in the current study, would be the initial step in the indirect pathway between food insecurity and youth adjustment, as caregiver depression often results in disrupted parenting characterized by greater conflict and less warmth or responsiveness in the caregiver-adolescent relationship, which then relates to more psychosocial maladjustment in youth (e.g., Hale et al. 2020). The current findings, however, did not support such a serial model of indirect pathways; rather, caregiver depression and caregiver-relationship quality acted as parallel indirect mechanisms through which greater household food insecurity predicted greater adolescent behavior problems.

The current findings call attention to the need to address food insecurity at the household level in order to fully mitigate potential negative effects on youth. Almost twice as many adult caregivers experience food insecurity compared to their children (Coleman-Jensen et al. 2020); if the stress associated with this experience has a negative effect on caregiver mental health and parenting or the parent-child relationship, then the number of children and adolescents facing potential adverse impacts of household food insecurity is much higher than simply the count of those youth experiencing food insecurity themselves. Typical interventions to address food insecurity for adolescents, such as free meals at school, target teens who are identified as food insecure themselves, which means that those who may be considered food secure may be going without critical support in the face of household food insecurity. In addition, such interventions focus primarily on the provision of food to address hunger, and thus do not address the psychosocial needs of adolescents living in food insecure households.

Adolescence is a period of increased vulnerability for the development, expression, or exacerbation of mental and behavioral health concerns due to rapid and significant neurological, physical, and cognitive changes, coupled with increased exposure to stressors that may trigger underlying diatheses or exceed coping capacities (Evans et al. 2018). The current findings demonstrate that entering adolescence with a history of household food insecurity may further increase this risk. Moreover, the detrimental impact of household food insecurity on caregiver mental health and caregiver-adolescent relationship quality not only potentially creates stress that may contribute to psychosocial maladjustment, but also compromises potential protective resources associated with resiliency such as parental support (Willemen et al. 2009).

The current findings have several implications for policy and health service providers. Routine screening for food insecurity at the household level should be a key a component of scheduled health maintenance visits throughout childhood and adolescence. Few pediatricians in high-need settings currently screen for food insecurity (Barnidge et al. 2017). However, such screenings can be easily integrated into current intake interviews using brief, validated measures with patient-centered wording, including the two-item Hunger Vital Sign (Hager et al. 2010). Likewise, educators could play a significant role by detecting when students may be experiencing food insecurity, especially among adolescents who may not take direct advantage of food support 
interventions due to stigma. The findings also underscore the urgent need to fund federal programs that enhance lowincome families' overall access to food, such as the Supplemental Nutritional Assistance Program (e.g., SNAP).

Most importantly, however, the current findings point to the need to assess food insecurity at the household or family level, rather than only at the individual level. Adolescents who may report being food secure themselves are still potentially at an elevated risk for psychosocial maladjustment if their family is experiencing, or has experienced in the past, food insecurity, given the cumulative impact of household food insecurity on caregiver mental health and parenting. The finding that food insecurity may operate on adolescent mental and behavioral health, in part, through caregiver depression and poor caregiver-adolescent relationship quality suggests that health and social service providers can further support families and identify potential risks for adolescent behavior problems by incorporating routine screening for these issues. This may, in turn, create opportunities to make necessary referrals to mental health providers who can assist adolescents and their families. On the other hand, the current findings also highlight the importance of assessing for household food insecurity among adolescents who are demonstrating elevated levels of internalizing (e.g., depression, anxiety, withdrawal) and externalizing (e.g., aggression, conduct problems) behavior. Mental health providers working with adolescents could potentially assist their families to connect with federal, state, and local resources to address food insecurity, as well as identify when parents are experiencing psychological distress due to financial hardship.

This study has important limitations to be noted. Given that the final sample on which data analysis was performed consisted of approximately $60 \%$ of the baseline sample, there are potential questions about the generalizability of these findings. For example, families were excluded from the current analyses when there were changes in caregiver residence or when different caregivers provided data across waves, raising the possibility that the variables that contributed to those transitions (e.g., abuse or neglect, caregiver mental health, change in housing) may have either augmented or mitigated the effects of household food insecurity on adolescent psychosocial adjustment. In addition, the baseline data for the current analysis was collected nearly two decades ago, raising questions about whether the findings would generalize to families and adolescents facing similar circumstances today. It is also important to acknowledge that a relatively small proportion $(15 \%)$ of the sample reported experiencing food insecurity. Although this proportion is consistent with the percentage of households with children classified as food insecure in 2000, the year data on food security was collected in the current study (Coleman-Jensen 2020), the rate of food insecurity among households with children has declined significantly in the intervening two decades (Coleman-Jensen 2020). As such, the current sample appears to be representative of the general population at the time data was collected but may not necessarily reflect the general population today. Moreover, the most recent data reported on current levels of household food security was collected prior to the arrival of the COVID-19 pandemic in the United States, and thus may not reflect the experience of food insecurity due to the economic impact of the pandemic (Coleman-Jensen 2020). The current results are likely to be most applicable to those living in urban settings in wealthy and industrialized countries who are experiencing household food insecurity and poverty; applications of the findings to families in lower income or less industrialized countries or in rural settings should be made with caution.

With respect to potential methodological limitations, an attempt to keep the time required for interviews to a reasonable length resulted in the use of a modified, eight-item measure of household food insecurity that may have not captured the full extent of the overall prevalence of household food insecurity in comparison with the 18-item HFSS, though research has shown the two scales to be highly correlated. In addition, baseline scores for all three mediators were included as covariates in order to strengthen the causal argument between wave 2 food insecurity and the three wave 2 mediators of interest. It is important to note, however, that the time at which these variables were measured slightly preceded the beginning of the 12-month span over which food insecurity was retroactively evaluated. This may potentially undermine causal interpretation, as the model did not account for any changes that may have occurred to wave 1 variables (including baseline values of mediators) in the interim. Despite attempts to strengthen causal arguments via temporal sequencing, it is also important to emphasize that evidence for the mediating role of caregiver depression and relationship quality does not completely support causation, as this study did not employ an experimental design. In addition, only two potential mediators of the relation between household food insecurity and adolescent behavior problems were examined, leaving many other intervening variables, including potential moderators, unmeasured and unrepresented in the model. Given the complexity of human behavior, and the broad array of intra- and interpersonal and structural variables likely associated with both household food insecurity and adolescent psychosocial adjustment, one should not assume that the observed relations in the current study fully capture the complete essence of the broad relation between food insecurity and behavioral problems.

Confidence in the conclusions from the current study is strengthened by the use of multiple informants (e.g., caregiver and adolescent) for different measures, thus reducing the risk 
that common methods and informant response style may have accounted for a large portion of the covariance observed in the models. Additionally, baseline values of outcome variables (including mediators) were adjusted for at each step of analysis. This strengthened the argument for a causal relationship between household food insecurity and subsequent caregiver depressive symptoms and caregiver-adolescent relationship quality, as well as for a causal relationship between both caregiver depressive symptoms and poor relationship quality and subsequent adolescent behavior problems. In addition, models were adjusted for a range of potential confounds beyond income that are typically associated with both food insecurity and adolescent behavior. Lastly, the primary findings were replicated using multiple alternative models, lending additional support to the finding that household food insecurity predicts adolescent behavior problems over time through its negative impact on caregiver depression and caregiver-adolescent relationship quality.

\section{Conclusion}

Despite the occurrence of food insecurity in millions of households with children under the age of 18 years, there is limited research concerning the direct and indirect effects of food insecurity on youth psychosocial adjustment, especially among adolescents who often do not participate in interventions designed to address hunger that are more readily available for younger children (e.g., SNAP and school-lunch programs). Consistent with the Family Stress Model, the current study sought to understand how household food insecurity might contribute to the development or exacerbation of behavior problems among adolescents, both directly and indirectly through its impact on caregiver depression and caregiver-adolescent relationship quality. The present study found that household food insecurity experience during pre-or early adolescence significantly predicts adolescent behavior problems over time. Moreover, this effect appears to reflect both the direct adverse effects of food insecurity on adolescent psychosocial functioning, as well as a stepwise series of indirect effects over time, in which household food insecurity predicts subsequent caregiver depression and poorer quality caregiver-adolescent relationships, which in turn predict emergent behavior problems years later. Such results emphasize the critical need to screen for household food insecurity at all scheduled health maintenance visits. In addition, the current findings suggests that clinicians, educators, and social service providers who work with low-income families should routinely assess for parental mental health concerns and dysfunctional parent-child relational patterns and make appropriate referrals to behavioral health providers when indicated as in order to support positive psychosocial adjustment in youth facing economic hardship.

Authors' Contributions B.A.K. proposed the primary theoretical conceptualization used within the study and led and coordinated the drafting and revision of the manuscript; D.W. secured the dataset, performed data analyses, and helped to draft the manuscript; M.F.S. consulted on statistical analysis and interpretation of data and helped to draft the manuscript. All authors read and approved the final manuscript.

Funding The Welfare, Children, and Families: A Three-City Study (grant number ICPSR 4701) was funded by a grant from the United States Department of Health and Human Services. National Institutes of Health. Eunice Kennedy Shriver National Institute of Child Health and Human Development (grant number R01HD036093) and supported by additional support from other foundations and federal agencies (see http://web.jhu.edu/threecitystudy/Funders/ for a complete list of funders)

Data Sharing and Declaration The datasets generated and/or analyzed during the current study are available from https://www.icpsr.umich. edu/web/DSDR/studies/4701.

\section{Compliance with Ethical Standards}

Conflict of Interest The authors declare that they have no conflict of interest.

Ethical Approval This study was performed in line with the principles of the 1964 Declaration of Helsinki. The WCFS study protocol was approved by the Institutional Review Board (IRB) at each of the universities and data collection firms involved in the project (Angel et al. 2012).

Informed Consent Informed consent was obtained from all participants included in the study.

Publisher's note Springer Nature remains neutral with regard to jurisdictional claims in published maps and institutional affiliations.

\section{References}

Abrain, M., Hague, M., Johal, L., Mathur, P., Nel, W., Rais, A., Sandhu, R., \& Sharma, S. (2013). Maturation of the adolescent brain. Neuropsychiatric Disease and Treatment, 9, 449-461. https://doi.org/10.2147/NDT.S39776.

Achenbach, T. M. (1991). Manual for the child behavior checklist/4-8 and 1991 profile. Burlington, VT: Department of Psychiatry, University of Vermont.

Achenbach, T. M. (1997). Manual for the young adult self-report and young adult behavior checklist. Burlington, VT: Department of Psychiatry, University of Vermont.

Achenbach, T. M., \& Rescorla, L. A. (2001). Manual for the ASEBA school-age forms \& profiles. Burlington, VT: University of Vermont.

Alaimo, K., Olson, C. M., \& Frongillo, E. A. (2002). Family food insufficiency, but not low family income, is positively associated with dysthymia and suicide symptoms in adolescents. The Journal of Nutrition, 132(4), 719-725. https://www.ncbi.nlm.nih.gov/ pubmed/11925467. 
Andreu, Y., Galdón, M. J., Dura, E., Ferrando, M., Murgui, S., García, A., \& Ibáñez, E. (2008). Psychometric properties of the Brief Symptoms Inventory-18 (BSI-18) in a Spanish sample of outpatients with psychiatric disorders. Psicothema, 20, 844-850.

Angel, R., Burton, L., Chase-Lansdale, P. L., Cherlin, A., \& Moffitt, R. (2012). Welfare, children, and families: a three-city study. ICPSR04701-v7. Ann Arbor, MI: Inter-university Consortium for Political and Social Research [distributor], 2012-10-04. https:// doi.org/10.3886/ICPSR04701.v7.

Armsden, G. C., \& Greenberg, M. T. (1987). The Inventory of Parent and Peer Attachment: Individual differences and their relationship to psychological well-being in adolescence. Journal of Youth and Adolescence, 16, 427-454. https://doi.org/10. 1007/BF02202939.

Barnidge, E., LaBarge, G., Krupsky, K., \& Arthur, J. (2017). Screening for food insecurity in pediatric clinical settings: opportunities and barriers. Journal of Community Health, 42, 51-57. https://doi.org/10.1007/s10900-016-0229-z.

Bickel, G., Nord, M., Price, C., Hamilton, W., \& Cook, J. (2000). Guide to measuring household food security. Alexandria, VA: Food and Nutrition Service, United States Department of Agriculture. https://hungerfreecommunities.org/wp-content/ uploads/2011/04/USDA-guide-to-measuring-food-security.pdf.

Bronte-Tinkew, J., Zaslow, M., Capps, R., Horowitz, A., \& McNamara, M. (2007). Food insecurity works through depression, parenting, and infant feeding to influence overweight and health in toddlers. The Journal of Nutrition, 137(9), 2160-2165. https:// doi.org/10.1093/jn/137.9.2160.

Brumariu, L. E., \& Kerns, K. A. (2010). Parent-child attachment and internalizing symptoms in childhood and adolescence: a review of empirical findings and future directions. Development and Psychopathology, 22(1), 177-203. https://doi.org/10.1017/ S0954579409990344.

Burchinal, M. R., Follmer, A., \& Bryant, D. M. (1996). The relations of maternal social support and family structure with maternal responsiveness and child outcomes among African American families. Developmental Psychology, 32(6), 1073-1083. https:// doi.org/10.1037/0012-1649.32.6.1073.

Coleman-Jensen, A., Rabbit, M. P., Gregory, C. A., \& Singh, A. (2020). Household food security in the United States in 2019. US Department of Agriculture, Economic Research Service. https://www.ers.usda.gov/webdocs/publications/99282/err-275. pdf? $\mathrm{v}=9458$.

Conger, R. D., Conger, K. J., \& Martin, M. J. (2010). Socioeconomic status, family processes, and individual development. Journal of Marriage and the Family, 72(3), 685-704. https://doi.org/10. 1111/j.1741-3737.2010.00725.x.

Cook, J. T., Frank, D. A., Berkowitz, C., Black, M. M., Casey, P. H., Cutts, D. B., \& Frank, D. A. (2013). Are food insecurity's health impacts underestimated in the U.S. population? Marginal food security also predicts adverse health outcomes in young U.S. children and mothers. Advances in Nutrition, 4(1), 51-61. https:// doi.org/10.3945/an.112.003228.

Daryani, I., Hamilton, J. L., Abramson, L. Y., \& Alloy, L. B. (2016). Single mother parenting and adolescent psychopathology. Journal of Abnormal Child Psychology, 44, 1411-1423. https://doi. org/10.1007/s10802-016-0128-x.

Derogatis, L. R. (2000). Brief symptom inventory (BSI)-18. Administration, scoring and procedures manual. Minneapolis, MN: NCS Pearson.

Evans, D., Borriello, G. A., \& Field, A. P. (2018). A review of the academic and psychological impact of the transition to secondary education. Frontiers in Psychology, 9, 1482 https://doi.org/10. 3389/fpsyg.2018.01482.

Fox, R. A., Platz, D. L., \& Bentley, K. S. (1995). Maternal factors related to parenting practices, developmental expectations, and perceptions of child behavior problems. Journal of Genetic Psychology, 156(4), 431-441. https://doi.org/10.1080/00221325. 1995.9914835.

Goodman, S. H., Rouse, M. H., Connell, A. M., Broth, M. R., Hall, C. M., \& Heyward, D. (2011). Maternal depression and child psychopathology: a meta-analytic review. Clinical Child and Family Psychology Review, 14, 1-27. https://doi.org/10.1007/s10567010-0080-1.

Hager, E. R., Quigg, A. M., Black, M. M., Coleman, S. M., Heeren, T., Rose-Jacobs, R., \& Frank, D. A. (2010). Development and validity of a 2 -item screen to identify families at risk for food insecurity. Pediatrics, 126(1), e26-e32. https://doi.org/10.1542/ peds.2009-3146.

Hale, W. W., Nelemans, S. A., Meeus, W. H. J., \& Branje, S. J. T. (2020). A 6-year longitudinal study of adolescents and mothers depression symptoms and their perception of support and conflict. Child Psychiatry and Human Development, 51, 407-415. https:// doi.org/10.1007/s10578-019-00952-y.

Hamilton, W. L., Cook, J. T., Thompson, W. W., Buron, L. F., Frongillo, E. A., Jr., Olson, C. M., \& Wehler, C. A. (1997). Household food insecurity in the United States in 1995: Summary Report of the Food Security Measurement Project. Food and Consumer Services, U.S. Department of Agriculture. https://www. fns.usda.gov/household-food-security-united-states-1995-summa ryreport-food-security-measurement-project.

Hardaway, C. R., \& Cornelius, M. D. (2014). Economic hardship and adolescent problem drinking: family processes as mediating influences. Journal of Youth and Adolescence, 43, 1191-1202. https://doi.org/10.1007/s10964-013-0063-x.

Harding, J. F. (2015). Increases in maternal education and low-income children's cognitive and behavioral outcomes. Developmental Psychology, 51(5), 583-599. https://doi.org/10.1037/a0038920.

Hayes, A. F. (2012). PROCESS: a versatile computational tool for observed variable mediation, moderation, and conditional process modeling [White paper]. https://www.afhayes.com/public/ process2012.pdf.

Hayes, A. F. (2018). Introduction to mediation, moderation, and conditional process analysis. 2nd ed. New York. NY: The Guilford Press.

Jyoti, D. F., Frongillo, E. A., \& Jones, S. J. (2005). Food insecurity affects school children's academic performance, weight gain, and social skills. The Journal of Nutrition, 135(12), 2831-2839. https://doi.org/10.1093/jn/135.12.2831.

Kerpelman, J. L., McElwain, A. D., Pittman, J. F., \& Adler-Baeder, F. M. (2016). Engagement in risky sexual behavior: adolescents' perceptions of self and the parent-child relationship matter. Youth and Society, 48(1), 101-125. https://doi.org/10.1177/ $0044118 X 13479614$.

Lee, C. S., Lee, J., \& August, G. J. (2011). Financial stress, parental depressive symptoms, parenting practices, and children externalizing problem behaviors: underlying processes. Family Relations, 60(4), 476-490. https://doi.org/10.1111/j.1741-3729.2011. 00656.x.

Marcus, R. F., \& Betzer, P. D. S. (1996). Attachment and antisocial behavior in early adolescence. The Journal of Early Adolescence, 16(2), 229-248. https://doi.org/10.1177/0272431696016002006.

McAdams, T. A., Rijsdijk, F. V., Narusyte, J., Ganiban, J. M., Reiss, D., Spotts, E., \& Eley, T. C. (2017). Associations between the parent-child relationship and adolescent self-worth: a genetically informed study of twin parents and their adolescent children. Journal of Child Psychology and Psychiatry, and Allied Disciplines, 58(1), 46-54. https://doi.org/10.1111/jcpp.12600.

McCleary, L. (2002). Using multiple imputation for analysis of incomplete data in clinical research. Nursing Research, 51(2), 339-343. https://doi.org/10.1097/00006199-20020900000012. 
Minh, A., Muhajarine, N., Janus, M., Brownell, M., \& Guhn, M. (2017). A review of neighborhood effects and early child development: how, where, and for whom, do neighborhoods matter? Health and Place, 46, 155-174. https://doi.org/10.1016/j. healthplace.2017.04.012.

Moffitt, R. A., \& Ribar, D. C. (2016). Child age and gender differences in food insecurity in a low-income inner-city population. National Bureau of Economic Research. https://www.nber.org/pa pers/w22988.

Munger, A. L., Hofferth, S. L., \& Grutzmacher, S. K. (2016). The role of the Supplemental Nutrition Assistance Program in the relationship between food insecurity and probability of maternal depression. Journal of Hunger and Environmental Nutrition, 11 (2), 147-161. https://doi.org/10.1080/19320248.2015.1045672.

Myers, A. M., \& Painter, M. A. (2017). Food insecurity in the United States of American: an examination of race/ethnicity and nativity. Food Security, 9, 1419-1432. https://doi.org/10.1007/s12571-017-0733-8.

Papini, D. R., \& Roggman, L. A. (1992). Adolescent perceived attachment to parents in relation to competence, depression, and anxiety: a longitudinal study. Journal of Early Adolescence, 12 (4), 420-440. https://doi.org/10.1177/0272431692012004005.

Poole-Di Salvo, E., Silver, E. J., \& Stein, R. E. (2016). Household food insecurity and mental health problems among adolescents: What do parents report? Academic Pediatrics, 16(1), 90-96. https://doi.org/10.1016/j.acap.2015.08.005.

Preacher, K. J., \& Hayes, A. F. (2008). Asymptotic and resampling strategies for assessing and comparing indirect effects in multiple mediator models. Behavior Research Methods, 40, 879-891. https://doi.org/10.3758/BRM.40.3.879.

Ramtekkar, U. P., Reierson, A. M., Todorov, A. A., \& Todd, R. D. (2010). Sex and age differences in Attention-Deficit/Hyperactivity Disorder symptoms and diagnoses: implications for DSM-V and ICD-11. Journal of the American Academy of Child and Adolescent Psychiatry, 49(3), 217-228. https://doi.org/10. 1016/j.jaac.2009.11.011.

Rose-Jacobs, R., Black, M. M., Casey, P. H., Cook, J. T., Cutts, D. B., Chilton, M., \& Frank, D. A. (2008). Household food insecurity: associations with at-risk infant and toddler development. Pediatrics, 121(1), 65-72. https://doi.org/10.1542/peds.2006-3717.

Ryan, R. M., Kalil, A., \& Leininger, L. (2009). Low-income mothers' private safety nets and children's socioemotional well-being. Journal of Marriage and Family, 71(2), 278-297. https://doi.org/ 10.1111/j.1741-3737.2008.00599.x.

Simons, L. G., Wickrama, K. A. S., Lee, T. K., Landers-Potts, M., Cutrona, C., \& Conger, R. D. (2016). Testing family stress and family investment explanations for conduct problems among African American adolescents. Journal of Marriage and Family, 78(2), 498-515. https://doi.org/10.1111/jomf.12278.

Slack, K. S., \& Yoo, J. (2005). Food hardship and child behavior problems among low-income children. Social Service Review, 79 (3), 511-536. https://doi.org/10.1086/430894.
Slopen, N., Fitzmaurice, G., Williams, D. R., \& Gilman, S. E. (2010). Poverty, food insecurity, and the behavior for childhood internalizing and externalizing disorders. Journal of the American Academy of Child and Adolescent Psychiatry, 49(5), 444-452. https://doi.org/10.1016/j.jaac.2010.01.018.

Tearne, J. E. (2015). Older maternal age and child behavioral and cognitive outcomes: a review of the literature. Fertility and Sterility, 103(6), 1381-1391. https://doi.org/10.1016/j.fertnstert. 2015.04.027.

United States Department of Agriculture (2018). Definitions of food security. https://www.ers.usda.gov/topics/food-nutrition-assista nce/food-security-in-the-us/definitions-of-food-security/.

Whitaker, R. C., Phillips, S. M., \& Orzol, S. M. (2006). Food insecurity and the risks of depression and anxiety in mothers and behavior problems in their preschool-aged children. Pediatrics, 118(3), e859-e868. https://doi.org/10.1542/peds. 2006-0239.

Whitsett, D., Sherman, M. F., \& Kotchick, B. A. (2019). Household food insecurity in early adolescence and risk of subsequent behavior problems: does a connection persist over time? Journal of Pediatric Psychology, 44(4), 478-489. https://doi.org/10.1093/ jpepsy/jsy088.

Willemen, A. M., Schuengel, C., \& Koot, H. M. (2009). Physiological regulation of stress in referred adolescents: the role of the parentadolescent relationship. Journal of Child Psychology and Psychiatry, 50(4), 482-490. https://doi.org/10.1111/j.1469-7610. 2008.01982.x.

Beth A. Kotchick is an Associate Professor at Loyola University Maryland. Her research interests include parenting and family processes, child and adolescent psychopathology, adolescent peer relationships, cognitive-behavioral theory and therapy with children and adolescents.

David Whitsett is a Licensed Clinical Psychologist at Western Youth Services in Orange County, California. His research interests include child and adolescent clinical psychology, pediatric psychology, family factors in behavioral health, and parenting.

Martin F. Sherman is Professor Emeritus at Loyola University Maryland. His research interests include psychopathology and personality, survey and attitude research, statistical methods, posttraumatic stress disorder (PTSD), and resilience. 